\title{
Discontinuous finite element numerical modelling for infrared thermographic crack characterization
}

\author{
by J. Rodríguez-Aseguinolaza*, J. González***, R. Celorrio***, A. Mendioroz* and A. Salazar*
}

\begin{abstract}
* Departamento de Física Aplicada I, Escuela de Ingeniería de Bilbao, Universidad del País Vasco UPV/EHU, Plaza Ingeniero Torres Quevedo 1, 48013 Bilbao, Spain, javier.rodriguezas@ehu.es

**Department of Applied Physics, CINVESTAV Unidad Mérida, carretera Antigua a Progreso km 6, A.P. 73 Cordemex, Mérida, Yucatán 97310, Mexico

***Departamento de Matemática Aplicada, EINA/IUMA, Universidad de Zaragoza, Campus Río Ebro, Edificio

Torres Quevedo, 50018 Zaragoza, Spain
\end{abstract}

\begin{abstract}
A new numerical model based on discontinuous Galerkin Finite Element Methods for laser spot lock-in thermopgraphy has been developed able to characterize very narrow cracks in materials, difficult to quantify by alternative numerical methods. It has also been validated by means of experimental results from a wide variety of cracks in terms of size, width, depth and crack orientation angle. Overall, the obtained results indicate a very good agreement with experiments maintaining a satisfactory accuracy and improved computational economy.
\end{abstract}

\section{Introduction}

In the last years, infrared thermography has attracted an increasing attention from both, the scientific and the technologic community in order to attain a mature technological development able to open new frontiers in the crack detection in materials [1]. The increasing complexity of the materials used in industries such as automotive, aerospace or metallurgy require the non destructive detection and full characterization of sub-micron and/or arbitrary shaped cracks, leading to severe experimental difficulties. Trying to overcome them, the use of computational modelling has been revealed as a very powerful method in order to complement experimental infrared thermography. As a result, the combined experimental-modelling based crack detection could be an appropriate solution, opening new application windows for generic crack detection. In this frame, different analytical and numerical models have been developed to obtain the surface or volume temperature distribution of the sample specimen under optical modulated excitation (figure 1a). Whereas the applicability of analytical approaches presents a very limited range, only valid for simple straight and infinite cracks, numerical approaches provide the needed flexibility and accuracy for a wider application frame.

Different numerical modelling strategies have been proposed in literature. Among the most used, continuous Galerkin (CG) finite element methods (FEM) have been proposed. This alternative leads to a continuous solution of the heat transfer equation on the complete domain (figure 1b), including the crack which requires very fine spatial discretizations in order to obtain the heat transfer perturbation derived from it. Even if successful solutions are found, these methods can be highly computationally expensive as the crack width decreases. With the goal of optimizing the calculation economy and extend the numerical treatment to very narrow cracks, hardly handled by CG-FEM, a new numerical method has been developed in this work, based on a Discontinuous Galerkin FEM approach [2] applied to laser spot lock-in thermography. This allows a weak formulation of the heat transport phenomena, enabling a non continuous thermal distribution over the crack, which is modelled as a single interface with a given thermal resistance $\left(R_{t h}\right)$ value (figure $\left.1 \mathrm{c}\right)$.
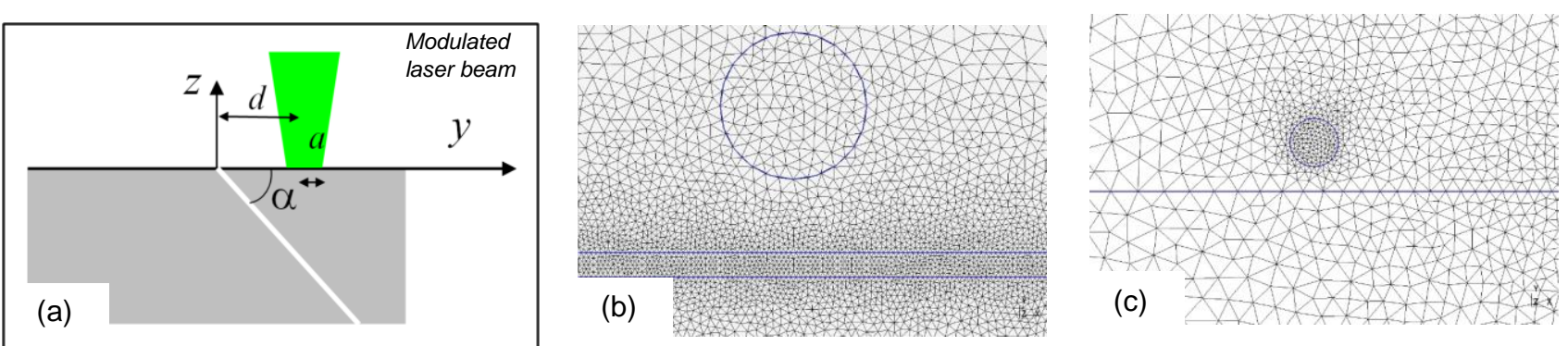

Fig. 1. (a) Schematic view of a modulated lock-in thermography experiment with laser spot excitation (green) (b) Continuous FEM spatial discretization. The blue circle represents the laser spot and the region between the two blue straight lines the crack. (c) Discontinuous Galerkin FEM discretization. The crack is a single interface. 


\section{Numerical model}

A numerical DG-FEM model has been developed in order to account for the steady-state thermal distribution in cracked specimens under modulated optical excitation. The heat transport constitutive equation (1) of the model sets an imaginary temperature field $\phi$ which provides the corresponding thermal amplitude and phase.

$$
\nabla^{2} \phi+i \frac{2 \pi f}{\alpha} \phi=0
$$

where $\phi$ stands for the specimen temperature field, $f$ is the optical excitation frequency (harmonic lock-in) and $\alpha$ is the thermal diffusivity of the sample. In order to obtain a closed set of equations, as shown in equation (2) Robin boundary condition is used, able to account for the modulated surface external heat supply and thermal losses.

$$
\left.\kappa \nabla \phi \cdot \hat{n}\right|_{S}+\gamma \phi=g
$$

where $\mathcal{K}$ is the thermal conductivity of the sample, $\hat{n}$ is the unit vector normal to sample surface, $\gamma=h_{\text {conv }}+h_{\text {rad }}$ accounts for the convective and radiative thermal losses and $g$ is related to the externally supplied surface power density. Considering the non-continuous nature of the modelled spatial domain by means of DG-FEM, additional heat flux conditions need to be imposed through the domain elements. In particular, the crack, which is assumed to be a single interface, requires a heat flux imposed by equation (3) together with a finite temperature jump given by a thermal resistance $R_{t h}=w / \kappa_{\text {air }}$, provided a crack with a width equal to $w$ filled with air with a thermal conductivity equal to $\kappa_{\text {air }}$, as shown in equation (4)

$$
\begin{gathered}
{\left.[[\kappa \nabla \phi \cdot \hat{n}]]\right|_{\text {Crack }}=0} \\
{[[\phi]]_{\text {Crack }}+\left.R_{t h}\{(\kappa \nabla \phi \cdot \hat{n})\}\right|_{\text {Crack }}=0}
\end{gathered}
$$

where [[ ]] stands for a finite jump between DG-FEM elements. The complete set of equations (1)-(4) are simultaneously solved by using the FEniCS open-source FEM computing platform.

\section{Model validation}

In order to guarantee the applicability range and accuracy of the developed model, different cracks have been experimentally characterized by means of laser spot lock-in thermopgraphy and compared with the obtained numerical results. Among them, vertical infinite, vertical finite in length and depth or slanted infinite cracks have been tested. The experimental results have been obtained emulating the crack by using rigid separation tapes of different width values between two material pieces. In all cases, as shown in figure 2, the experimental and numerical agreement is very good.

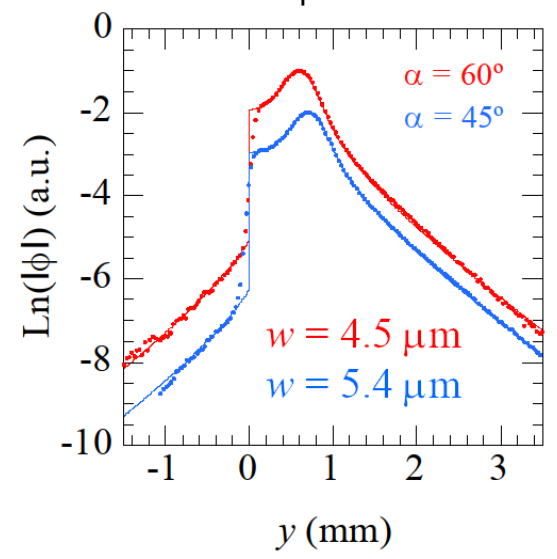

In conclusion, the developed numerical model offers a validated accuracy on the modelling of a wide range of very narrow crack typologies in terms of crack width, angle and depth with a noticeable computational economy, difficult to attain with other FEM methods.

This work has been supported by Ministerio de Economía y Competitividad, (DPI2016-77719-R, AEI/FEDER, UE), by Universidad del País Vasco UPV/EHU (GIU16/33) and by Gobierno Vasco (PIBA 2018-15).

Fig. 2. Comparison between experimental (dots) and numerical (lines) results for an AISI-304 steel sample with slanted cracks at $45^{\circ}$ and $60^{\circ}$ and a width of around $w=5 \mu \mathrm{m}$. The longitudinal ( $y$-figure 1a) logarithmic temperature distribution obtained with a lock-in frequency $=1.5 \mathrm{~Hz}$ is shown.

\section{REFERENCES}

[1] Li T., Almond D.P., Rees D.A.S., "Crack imaging by scanning laser-line thermography and laser-spot thermography" Measurement Science \& Technology 22, 035701 (2011)

[2] Celorrio R., Omella A.J., Pech-May N.W., Oleaga A., Mendioroz A., Salazar A., "Vertical cracks characterization using lock-in thermography: II. Finite cracks". Measurement Science \& Technology 25, 115602 (2014) 